\title{
Analysis of Beneficial Bacterial Populations from Chinese Longbeans
}

Alexis Zaide, Jeff Hillyer, Scott Holt*, Western Illinois University, Biology, Macomb, IL

\section{Specific Goals}

understand the biodiversity of natural beneficial plant bacteria

build a collection of beneficial bacteria \& assess their effectiveness for boosting plant growth

harness microbial biodiversity for development of natural agricultural biologicals that support the growth of crop plants

Scott Holt

sm-holt@wiu.edu 


\section{Analysis of Beneficial Bacterial Populations from Chinese Longbeans}

Alexis Zaide, Jeff Hillyer, Scott Holt*, Western Illinois University, Biology, Macomb, IL

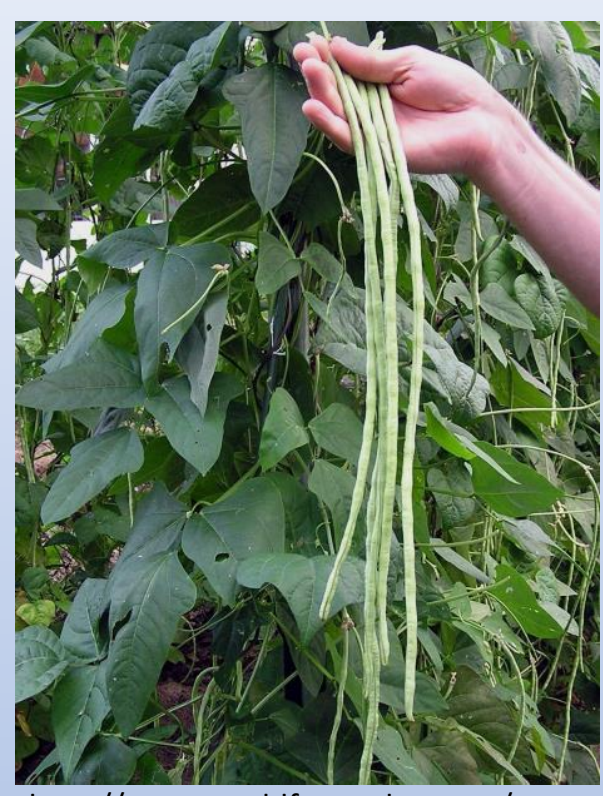

https://www.tbo.com/storyimage/T B/20131023/ARTICLE/131029832/E P/1/2/EP-131029832.jpg

htp://www.worldformarket.com/w -content/uploads/2016/02/YardBean.gif

https://petalsandwings.files.w ordpress.com/2012/08/longb ean-7-1.jpg

\section{USDA Plant Guide}

Food Source

pods, young leaves, \& stem tips edible

humans \& wildlife (Deer)

Commercial

harvested sold farmers markets \& local distributers

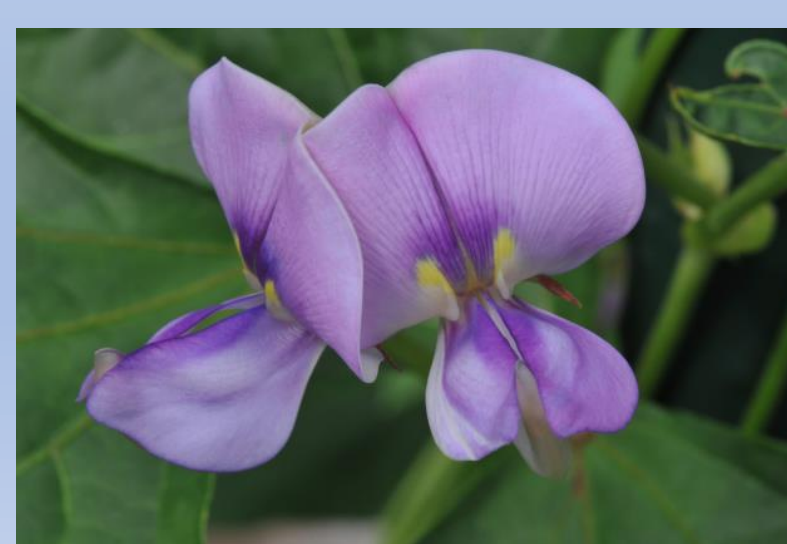

\section{Ornamental}

large violet-blue flowers \& draping pods useful city parks, office buildings, homes 
Analysis of Beneficial Bacterial Populations from Chinese Longbeans

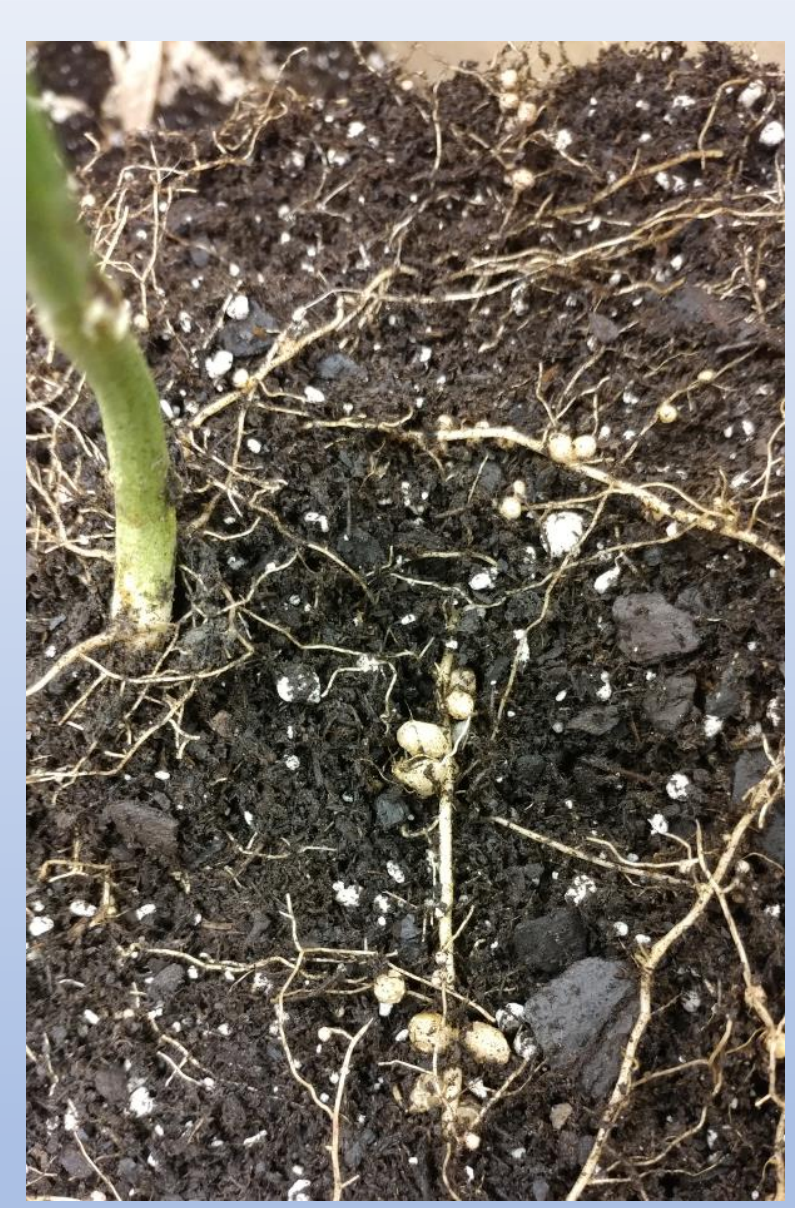

\section{Project Goals}

assess the beneficial bacterial populations on longbeans leaves \& nodules

use culture dependent \& culture independent methods



\section{Leaves}

Methylobacterium species beneficial for plants

\section{Nodules}

symbiotic rhizobia spp infect root nodules

i.e. = Bradyrhizobium? 
Analysis of Beneficial Bacterial Populations from Chinese Longbeans



Stroma $(100 \mathrm{~s} / \mathrm{mm})$
Leaves: Methylobacterium species

gram negative, rod shaped cells

live on plant leaf surfaces

metabolize $\mathrm{C} 1$ = methanol (dehydrogenase)

liberated from plant during growth

Significance $=$ Provide Benefits to Many Plants improve seed germination \& crop yield,

pathogen resistance \& drought stress tolerance

excrete plant growth hormones \& osmoprotectants positively influence plant growth \& health. used as natural seed inoculates by ag biotech co. 
Analysis of Beneficial Bacterial Populations from Chinese Longbeans Experimental Outline

Leaves (Methylobacterium) or Nodule (rhizobia)

Culture Dependent

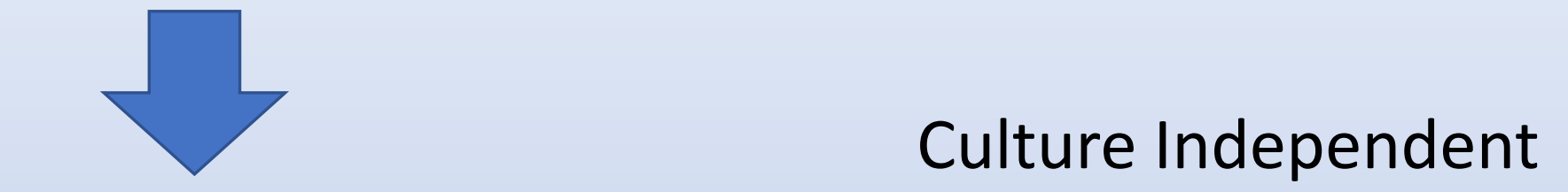

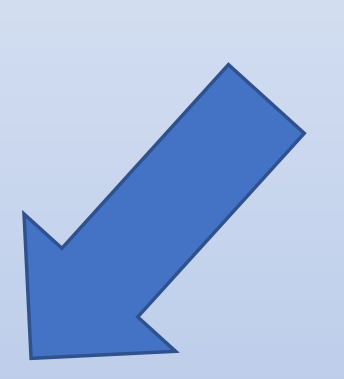

1. Total Bacterial Count

2. Methylobacterium Count

3. Rhizobia count (nodule)

Culture Collection

Store at $-80^{\circ} \mathrm{C}$

\section{Extracts}

Leaves \& Nodules

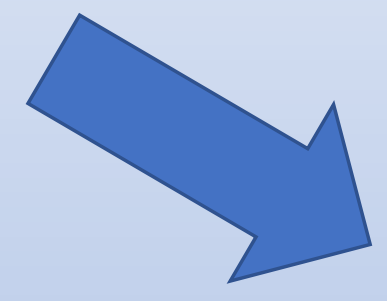

Microbial DNA

PCR

$16 S$ rDNA Amplicons

Sequencing

Main Bacterial Phyla Proportion to each other 


\section{Composition of Beneficial Bacterial Populations on Plant Species}

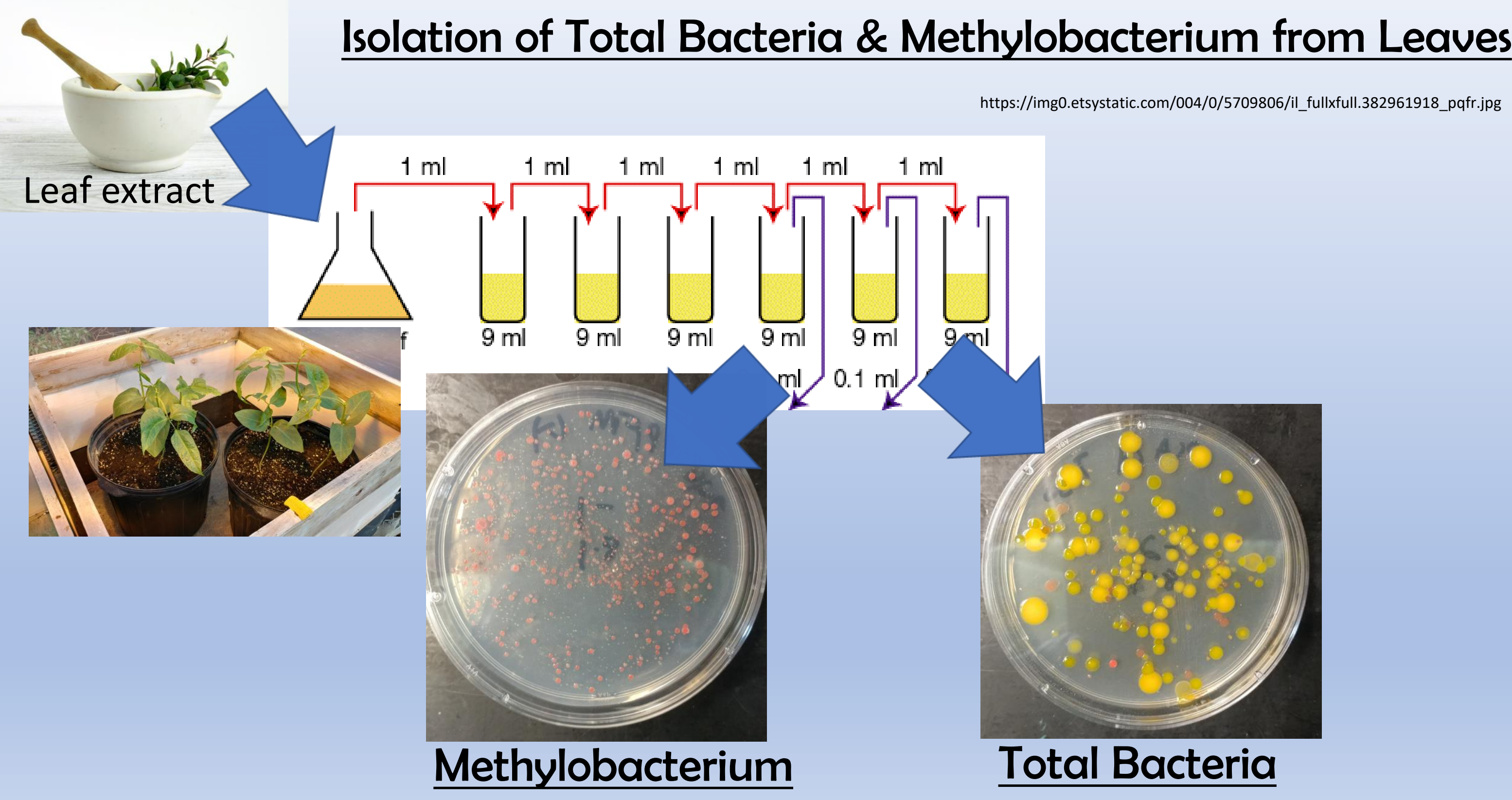


Pilot Study: Total Aerobic Bacterial Viable Count on Longbean Leaves

Greenhouse Beans Vs Garden Beans

Total Aerobic Bacterial Viable Count

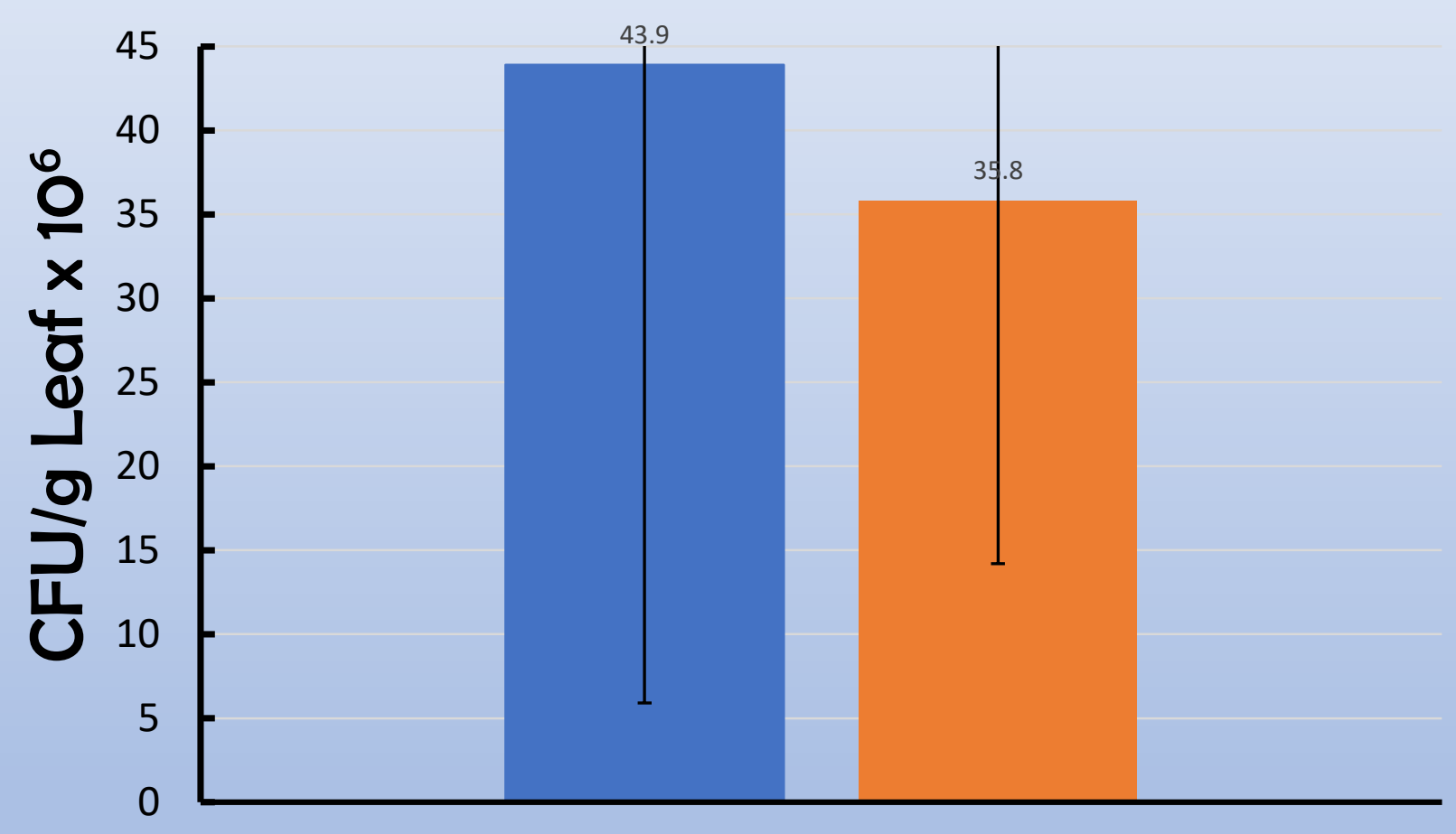

Greenhouse Garden

mean value from 3 leaves

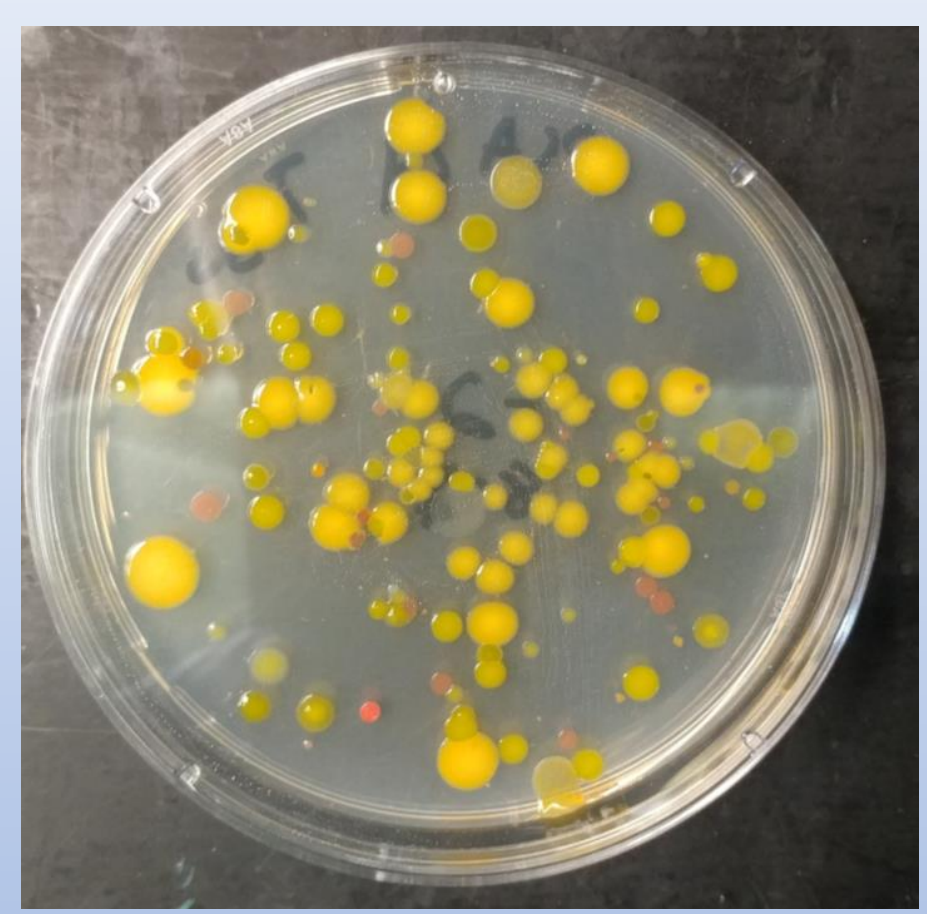

Heterotrophic Bacterial Count Plate Count Agar

() tremendous variability ()) no significant difference 


\section{Pilot Study: Total Methylobacterium Viable Count on Longbean Leaves}

\section{Greenhouse Beans Vs Garden Beans}

Pink Pigmented Facultative Methylotroph Viable Count

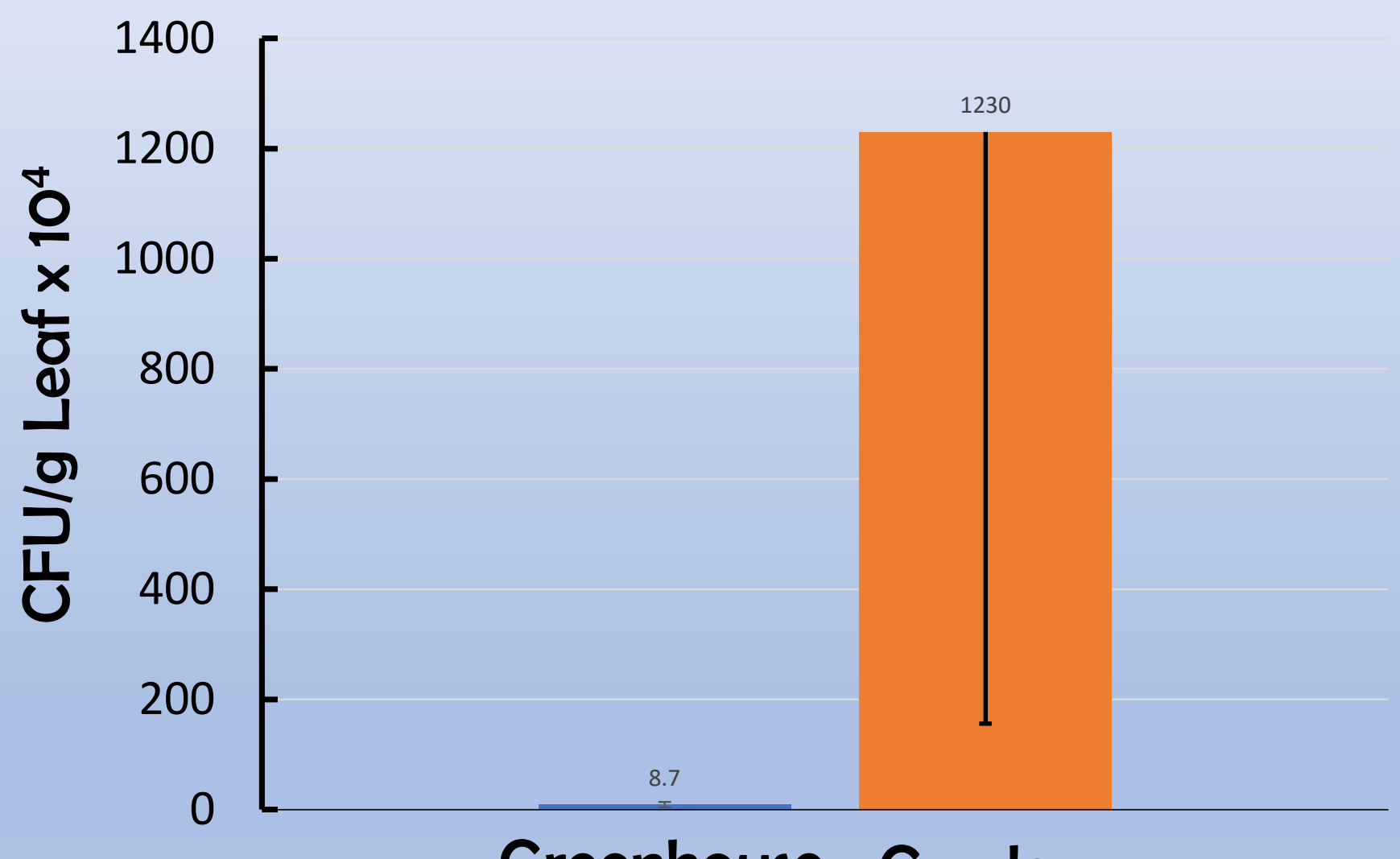

Greenhouse Garden

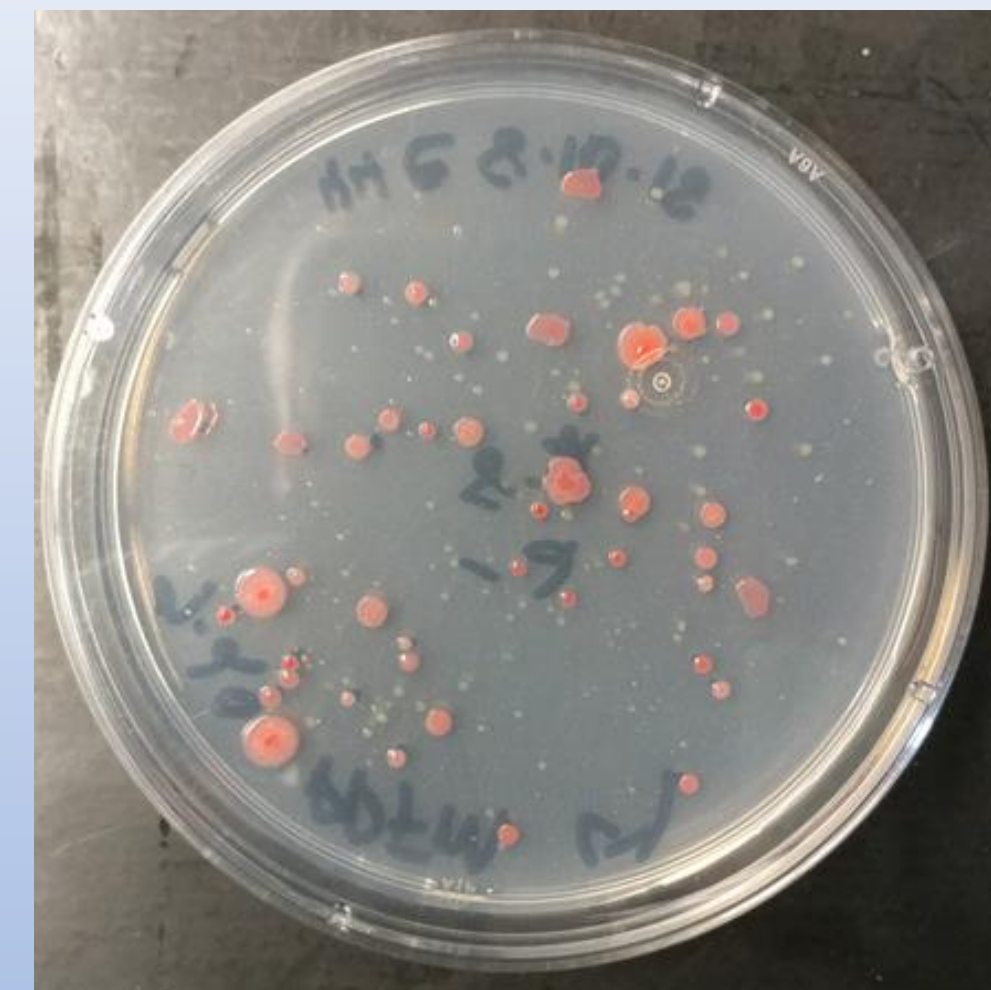

Methylobacterium on AMS w/ methanol Pink colonies

() tremendous variability ;) no significant difference 
Analysis of Beneficial Bacterial Populations from Chinese Longbeans

rhizobia viable count from root nodules

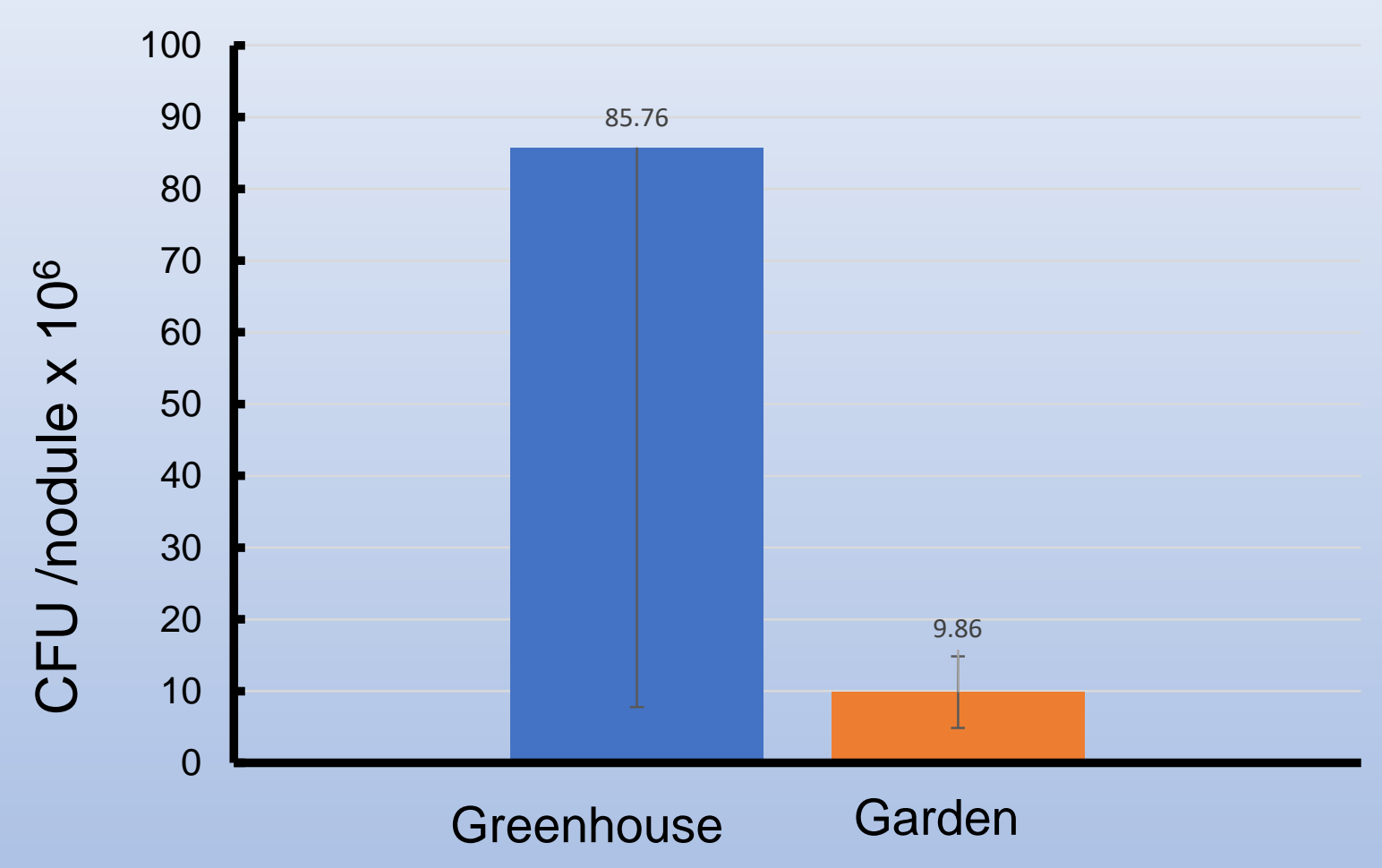

(-) tremendous variability

()) no significant difference

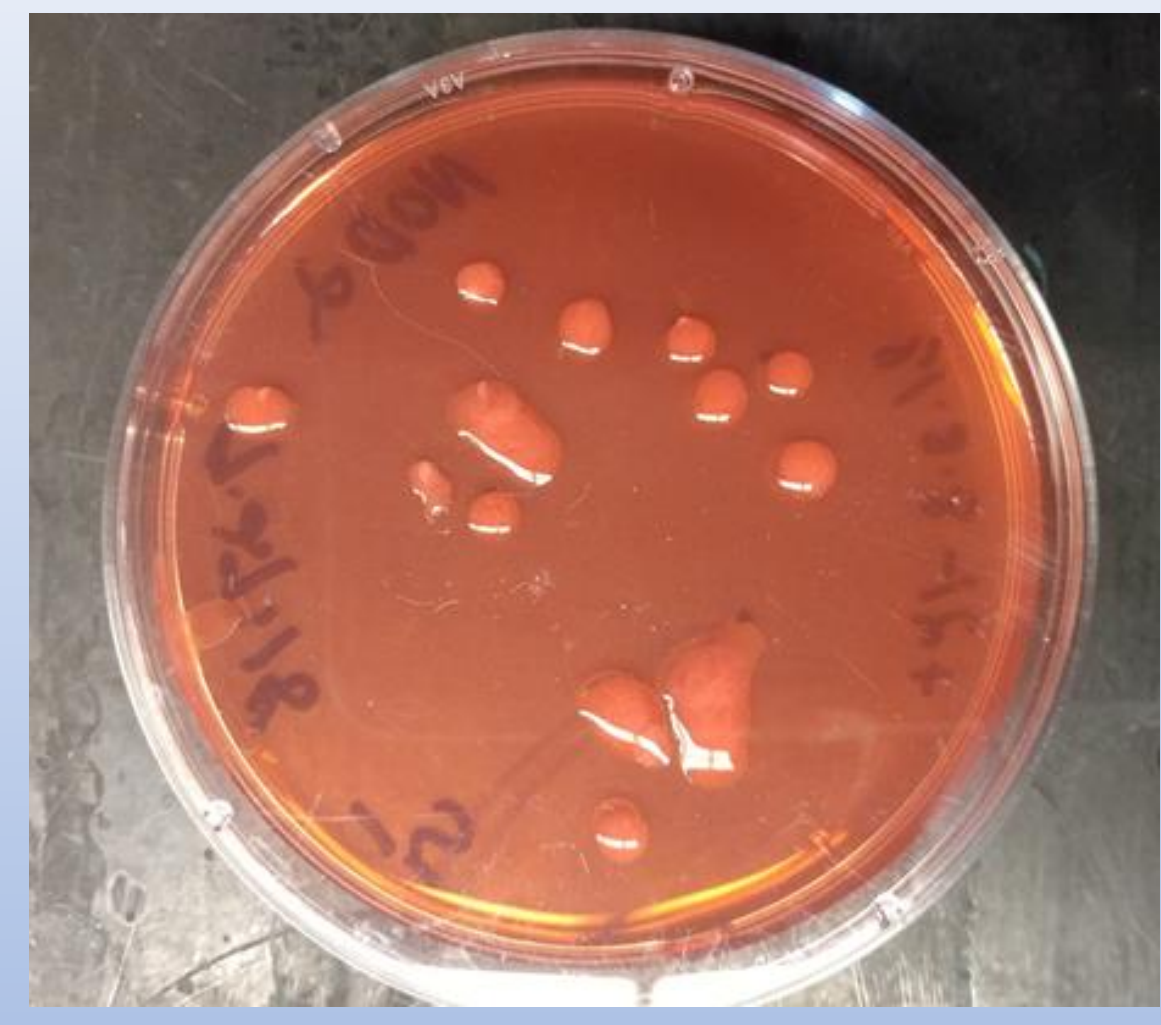

Nodule Rhizobia on CRYMA

2 weeks incubation @ room temperature CFU / mg better than CFU / nodule? 
Analysis of Beneficial Bacterial Populations from Chinese Longbeans

\begin{tabular}{|l|l|}
\hline Table 1. Identification of Rhizobia from root nodules \\
\hline Cultural Characteristics & $\begin{array}{l}\text { cream or pinkish, mucoid colonies } \\
\text { 14 days incubation on *CRYMA }\end{array}$ \\
\hline Cellular Characteristics & $\begin{array}{l}\text { Gram negative, rod-shaped } \\
\text { granules of poly-B-hydroxybutyrate }\end{array}$ \\
\hline Molecular (16S V4 region) & $\begin{array}{l}\text { "Bradyrhizobium" typical (B. elkanii) } \\
\text { "Bradyrhizobium" atypical } \\
\text { (B. japonicum or B. radiobacter) }\end{array}$ \\
\hline
\end{tabular}

\section{Table 1 Summary}

Two types of Bradyrhizobium spp. were isolated from nodules but only one sp. type per nodule.

Bradyrhizobium "typical" (elkanii) was found in a majority of nodules. Bradyrhizobium "atypical" (japonicum) was found in an occasional nodule. 
Analysis of Beneficial Bacterial Populations from Chinese Longbeans

Table 2. Identification of Methylobacterium from Leaves

\begin{tabular}{l|l}
\hline Cultural Characteristics & pink-pigmented colonies
\end{tabular}

5-7 days grown on *AMS

\begin{tabular}{l|l}
\hline Cellular Characteristics & Gram negative, rod-shaped
\end{tabular}

lipid inclusions or volutin granules

Molecular (16S V4 region)

"Methylobacterium"

(extorquens or populi)

Table 2 Summary

Methylobacterium species were detected on leaves

Larger sequencing length needed for species 
Analysis of Beneficial Bacterial Populations from Chinese Longbeans Alexis Zaide, Jeff Hillyer, Scott Holt*, Western Illinois University, Biology, Macomb, IL

\section{Project Results Summary}

This is the first report on detection and identification of beneficial Methylobacterium and Bradyrhizobium spp. associated with the productive alternative food crop Chinese Longbeans.

It is also unusual to find two different rhizobia species form a symbiosis with the same crop plant.

The beneficial bacterial species isolated from productive Longbeans may be used to develop natural microbial inoculants that support the growth of other crop plants. 